\title{
USING THE FRAMEWORK OF DISTRIBUTED COGNITION IN THE EVALUATION OF A COLLABORATIVE TOOL
}

\author{
N. Hussain ${ }^{a}$, O. de Bruijn ${ }^{b}$ and Z. Hassan ${ }^{c}$ \\ a,bSchool of Informatics, The University of Manchester, United Kingdom, \\ email: an.hussain@postgrad.manchester.ac.uk

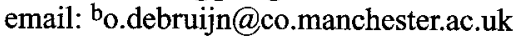 \\ c College of Information Technology, University Tenaga Nasional, Malaysia \\ email: zainuddin@uniten.edu.my
}

Abstract - This paper reports on the preliminary findings of an on-going research on the usability of an interactive tabletop, DiamondTouch in a computer-supported collaborative design environment. It summarizes the analysis of the collaborative design activities around the table. Modelled after a household coffee table, DiamondTouch provides users with an informal environment where users can sit on comfortable chairs while interacting with the table concurrently. The framework of distributed cognition was used as an analytic tool which demonstrated how the communicative events around the tabletop were used to bring the design representations into coordination with one another and how information processing activity was performed. Researcher examined the relationship between actors, artifacts, and the settings in which interactions occur. Within a single conceptual framework, the representations flowing through functional systems as objects of analysis could be identified and allowed researcher to reason about design artifacts and cognitive artifacts. These helped in identifying characteristics which could enhance the usability of DiamondTouch, and potential weaknesses of the tabletop.

Keywords: Collaborative design, distributed cognition, interactive tabletop

\section{INTRODUCTION}

To meet the challenges of ubiquitous computing, researchers are now exploring new approaches in the design of information technology to support co-located computer supported collaborative work (CSCW). Research into CSCW seeks to understand how people and organizations interact with one another, and to integrate this understanding with the development of computer based tools to support real world settings (Perry, 1997). In a collaborative environment such as design, people actively communicating and working together to establish design goals, search through design problem spaces, determine design constraints, and construct a design solution (Hennessy \& Murphy, 1999). Design workers need to work together on problem- 
solving task by questioning each other, and discussing and sharing information. These interactions are crucial in determining the final outcome of the design process. Depending on the nature of the design tasks, the participants usually use a wide range of tools and other organizing resources. The change of workflow that comes with the use of collaborative tools makes evaluation of such task very challenging for researchers. In addition, much group design activity is inherently cognitive where designers need to think, predict or make decisions. Therefore, there needs to be an understanding of how activities are performed at this level of analysis to ensure effective design of computer system that can support both cognitive activities and social interactions (Rogers \& Ellis, 1994). To date, there are only a few well-accepted, inexpensive methods for evaluating collaborative systems. It is even more challenging as many approaches such as the heuristic or expert reviews which are used effectively for single user interfaces do not take into account the complex interactions between individuals (Damianos et al., 1999). These approaches do not present adequate means of evaluating the dynamics of collaborative activity by neglecting the cognitive capabilities of the actors within the environment (Rogers \& Ellis, 1994).

On the other hand, the distributed cognition approach proposed by Hutchins (1990) is an ideal method to use to discover the social and cultural dimensions of collaborative design. This approach was developed to understand cognitive activities within workplace settings. Unlike traditional cognitive theory, distributed cognition extends the reach of what is considered cognitive beyond the individual to include interactions between people and other artifacts in the environment. A process is not noncognitive simply because it happens in the interactions among many brains, nor is cognitive simply because it happens in a brain (Hollan et al., 2000). This framework focuses on the representations involved in information processing because access to the representations involved in activity allows study to determine the resources used in the performance of problem solving and consequently, design (Perry, 1997). Artifacts such as interactive tabletops are the physical embodiments of representations, and the media through which representations are operated upon in the collaborative work. Distributed cognition has been used in many analyses to examine the cognitive properties of collaborative works. Among them are analyses of airline cockpits, air traffic control operations, the navigation systems of naval vessels, and collaboration between programmers (ibid). This method of analysis helps in identifying how such systems act as processors of information and how novel technology could be designed to both benefit work and implemented without disrupting activity through removing the resources used in co-ordination (Halverson, 1994). 
Research conducted by Perry (1997) revealed that the framework of distributed cognition can be used effectively in the development of computer supported co-operative work technologies. His study focused on the resources in collaborative design activities and discovered that a number of design processes can only be examined from a perspective which includes the social dimension of work. Using the distributed cognition approach, Perry identified novel technological infrastructures and configurations to support organizational and social process, organizational and inter-organizational activity, the flow of design, the development of technical memory, the coordination of spatially distributed collaboration, and meetings. Measuring how well a technology support these infrastructures and configurations could be used as an indication of the quality of it.

Drawing from the findings by Perry (ibid), the researcher adapted the distributed cognition approach in the evaluation of an interactive tabletop in a collaborative design environment. In traditional design settings, design team members would sit together to discuss design issues and sketch preliminary design that can then be carried out in detail by other members after the meeting. The discussions usually take place around a conference table. This face-to-face working environment allows one to keep track of what others are doing through a wide variety of perceptual cues. This led us to select the interactive tabletop, DiamondTouch (Deitz \& Leigh, 2001), as the collaborative tool in our study. Unlike other technology, i.e. digital whiteboard, an interactive tabletop takes advantage of both the affordance of physical tabletops to facilitate small-group collaboration and the experience people have collaborating around tables. The open workspace provided by a digital tabletop facilitates interaction, which is important for workspace awareness and coordination (Hutchins, 1995). Study by Gutwin and Greenberg (1999) revealed that awareness-enhanced interface can improve groupware usability. The awareness of others in the workspace involves knowledge of who is present, where they are working, and what they are doing. It is used in collaboration to coordinate activity, to simplify verbal communication, to provide appropriate assistance, and to manage movement between individual and shared work. Earlier study conducted by the researcher (Hussain et al., 2005) has also revealed that an interactive tabletop has the potential to support effective group collaborative design activities.

This report is part of an ongoing analysis on the coordination of the design process. It summarizes the communication and co-ordination, the distributed computation and collaboration, the structure of informational resources, the division of labor, and the role of context during the collaborative activities. Before presenting our findings, we give an overview of other available methods for evaluating collaborative system. 


\subsection{Evaluating collaborative systems}

Activity theory is an alternative cognitive approach originating in Soviet psychology in 1920s, aimed to understand the unity of activity and consciousness (Nardi, 1995). "Activity" refers to human interaction with the objective reality while "consciousness" refers to the human mind as a whole. This theory is concerned with practice or doing activity involving external devices and tools. It proposes that when artifacts are integrated into social practice, an activity cannot be understood without understanding the role of artifacts in every day existence. The theory also states that the human mind emerges and exists as a special part of human interaction with the environment. Therefore, the mind can be analyzed and understood only within the context of activity. With this theory, the analysis of a collaborative system incorporates strong notions of intentionality, history, mediation, collaboration and development in constructing consciousness.

The Collaboration Usability Analysis (CUA) is a modelling technique focuses on the teamwork aspects of a collaborative activity (Pinelle et al, 2004). It helps evaluators remember and test the range of uses that their collaborative system will see in a real situation by capturing the breadth and variability inherent in a group task. Evaluators can also consider how specific parts of the collaborative system interface support collaborative operations of communication and coordination using a set of group work primitives called the mechanics of collaboration. This modelling technique was developed as a framework for evaluators to simulate the realistic use of a groupware system and to identify usability problems that are caused by groupware interface.

\section{METHOD}

\subsection{Participants}

Thirty college students whom were referred as the designers, volunteered to take part in this experiment which was conducted in a laboratory environment. Each designer was assigned to a group of three which gave a total of 10 groups participated in the evaluation. In order to promote interactions, the designers were encouraged to select their own group members. All designers have a minimum of 5 years of experience using computers and less than 1 year of experience using touch screen displays. They were students enrolled in the Information Technology degree program and have no experience in house designs.

\subsection{Interface}

DiamondTouch is a multi-user touch technology for tabletop front-projected displays. It is modelled after a household coffee table, which provides users with an 
informal environment where users can sit on comfortable chairs while interacting with the table concurrently. Users can use its touch-surface simultaneously and independently from one another. The table-surface associates each touch on the surface with a particular user seated around it. In this environment people can easily shift their attention between the tabletop and other sources of information while having coffee and a chat with some friends. Applications can be developed to run on this tabletop that allow natural interactions at a physical table such as reorienting objects, passing documents around the table, and spreading and making a pile of documents. The designers perform their collaborative task around this tabletop. Besides DiamondTouch, personal computers were allocated to each designer in order for them to perform their individual tasks.

\subsection{Procedure}

The most realistic way of evaluating a system is to go into the place of work and to watch real users using it. However, the laboratory experiment method was used instead due to the complexity of a real design environment and the long period required for the observation. The evaluation of the tabletop involved the flow of design, the development of technical memory, the coordination of task, and meetings processes around the tabletop. Specifically, the researcher focused on the emergence of design solutions, the formal properties of media of representation, the communication and coordination of actions among the designers. Researcher examined how the enacted processes and the physical representations influenced the design task. These activities pre-specified the representational media and shaped the information processing activities that are applied in subsequent problem resolution (Perry, 1997). The framework of distributed cognition as discussed by Perry (ibid) was applied in the analysis which has guided the researcher in analyzing the elements of work system.

The experiment began with the designers in each group signing the consent forms. Designers were then briefed on the purpose and format of the experiment. A brief demonstration by the researcher on the features of the tabletop was also conducted and the designers were given ten minutes to explore DiamondTouch on their own.

Following this, the Subject Task Form was handed out. The Subject Task Form described the instructions and the tasks that need to be done by the designers. The designers were allowed to discuss among themselves for ten minutes on the task to be performed. Each group was required to produce a plan of a house that includes the $1^{\text {st }}$ floor, $2^{\text {nd }}$ floor, and the front view of the house. Each designer drew a different layout ( $1^{\text {st }}$ floor, $2^{\text {nd }}$ floor, or front view) of the house which they created individually using Microsoft Paint on separate personal computers (Figure 1). 


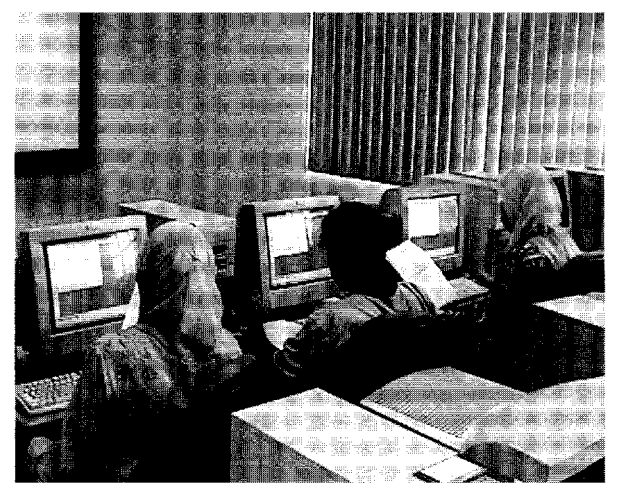

Figure 1 : Designers creating individual drawings

At this stage, the designers were not allowed to communicate with each other while creating the drawings. The personal computers were connected to the DiamondTouch. Upon completion of the drawings, the designers moved and sat around the tabletop. They retrieved their files at the tabletop and discussed their individual design with the other members of the group (Figure 2). The designers could retrieve their previous and current drawings for discussion and made temporary annotations. The annotations were only for discussion around the tabletop and were not stored permanently in the drawing files. Based on the feedbacks from the other members, the designers would proceed back to the personal computers and improve their drawings. They were allowed to discuss and update their drawings up to three (3) times and the time allocated for the task was sixty (60) minutes. At the end of the experiment, questionnaires were administered to gather feedbacks on the participants' experiences using DiamondTouch. The interactions around the tabletop were also videotaped for analysis. The following section summarizes the qualitative analysis of the videotapes using the distributed cognition approach.

\section{ANALYSIS}

\subsection{Communication and co-ordination}

The scenario of the evaluation required the designers to communicate with each other to solve the task. The main form of communication between the designers was through spoken language. The spoken language during the discussion around the tabletop brought the mentally held representational structures of the different designers into coordination with each other. 


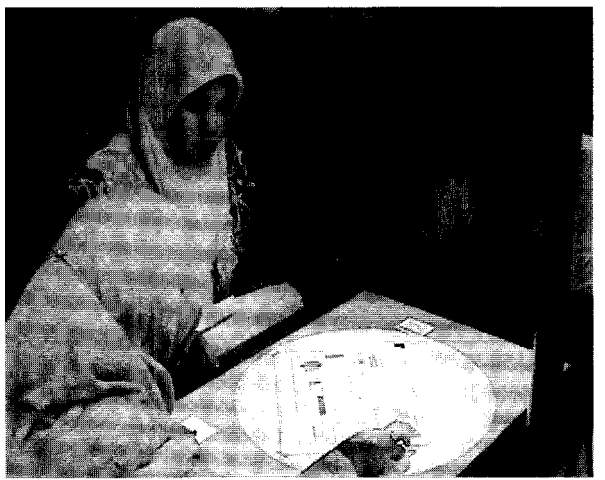

Figure 2 : Designers discussing their individual drawings

The designers were observed to discuss one layout of the drawings at a time (Figure 3). This promoted a lot of turn-taking where designers took turn in explaining their design. In some circumstances, the spoken language failed to coordinate activity due to its ambiguity, modality of the design, or lost of words (i.e. difficulties in finding the appropriate words to describe the designs) by the designers. This caused other method of communication chosen which was using the pen and paper.

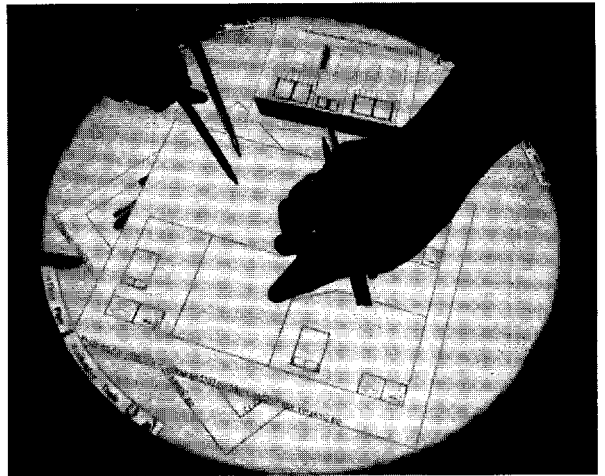

Figure 3 : Designers discussing one layout of the drawings at a time

The actions of the other participant using hand gestures also facilitated a common understanding because of the common background knowledge on the design task. These communicative events around the tabletop were used to bring cognitive design representations into coordination with one another. The production 
of the final design depends on the willingness of each designer to constrain their behavior to fit in with that of other designers, the constraints created by the nature of the tabletop and the personal computer, and the sequential constraints of the procedures they must follow as outlined in the task form. These constraints specify the social organization of the design process so that coordination is possible.

\subsection{Distributed computation and collaboration}

The representational artifacts used in the processes ranged from the list of design specifications given by the researcher, to basic pen and paper sketches, to the use of the drawing tool, and to the use of the DiamondTouch. Through these artifacts, the design representation were propagated and re-represented in different media.

Before the evaluation began, each designer was given a task form which listed the design specifications and task to be conducted. The list of design specifications served as a formal guideline or plan to produce the design output. This plan helps in the allocation of responsibilities among the designers. It was observed that the drawings were used explicitly as a medium for communication to transmit easily misunderstood information between individuals having different perspective and understandings about the design. The sketches on the drawing were done on the task forms itself. This demonstrated that the sketches were transitory media and were not intended as fully comprehensive design representations. Sketches on the paper were then used as a guide to create or update the drawings in Microsoft Paint. The DiamondTouch served as a common representational artifact in the setting. All the designers gathered around the tabletop to discuss their drawings. The size of the tabletop ( $80 \mathrm{~cm}$ diagonal) and the seating orientation promoted eye contacts among the designers while talking. All the designers easily reached and concurrently gestured towards the displayed images with their fingers. They externalized their individual's internal cognitive processes through the drawings and sketches so that the design representation propagations were available to the other group members (Perry, 1997).

\subsection{Structure of informational resources}

The resources that the designers used to structure their activities were the context of the task, the other designers involved in the task, and the individuals' personal cognitive world. The context of the task is a critical resource for action since it determines the access to physical resources (ibid). Activities were made explicit by mechanisms that included the used of tabletop, sitting positions, and features of the prototype application. The network connection between the personal computers and DiamondTouch facilitated the design processes by linking the design artifact to its use in communication and coordination among designers. The tools were not used 
in isolation but in combination with other tools through out the process. This setting was important to support the representational flow and to demonstrate how the representation in one medium could be easily transformed onto another medium. During the discussion around the tabletop, the designers assisted each other's task by providing suggestions, questions, or comments on the drawings through spoken language, annotation, or sketches on paper. The other important resource used was the cognition within individual. This resource, though not analyzed, was understood to be an important resource for coordinating the design work.

\subsection{The division of labor}

The division of labor determines the computational architecture of the problem solving unit (ibid). The design tasks were allocated through negotiated division of labor. When the task forms were handed out to the designers, they negotiated on the layout that they wanted to work on. This help to optimize and control the work processes. However, even though the designers would draw the layout independently using their personal computer, the designers sought and exchanged opinions among each others on how to improve and to complete the design during the discussion around the tabletop. Through the discussion, the individual drawings were reintegrated and consolidated with the other design as a whole. This was to ensure the separate drawings remain compatible with one another despite any changes. For example, as produced by one of the groups, the position of the staircase on the $1^{\text {st }}$ floor (Figure 4) had to match the position on the $2^{\text {nd }}$ floor (Figure 5) and the positions of some of the windows and doors had to match the front view (Figure 6).

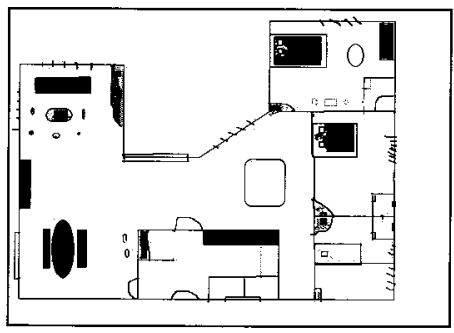

Figure $4: 1^{\text {st }}$ Floor

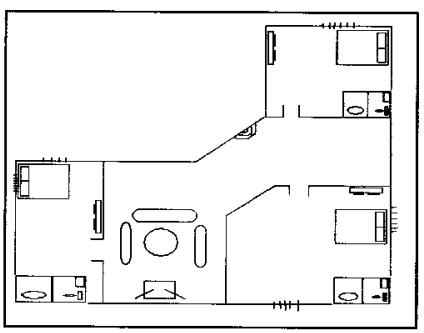

Figure 5: $2^{\text {nd }}$ Floor

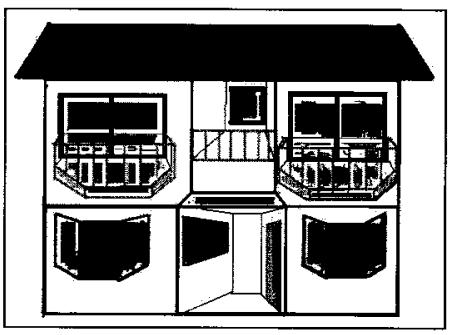

Figure 6 : Front View 


\subsection{The role of context}

The behavior of the designers was mainly constrained by the features provided by the prototype, the input device available for the tabletop, the size of the tabletop, and the time allocated to complete the design task. These served as the context of the activity which determined how the representation was carried, and how it could be transformed.

With the use of DiamondTouch, the coordination activities were generated on an ongoing basis and in a natural, unobtrusive way. It was used extensively to anchor discussion and provide shared understanding of the context. The tabletop enables shifting between seeing the representation and the thing that is represented. There was no global script or systematic plan that controlled these activities. The input device for the tabletop was only through the touch of fingers which allowed flexible interactions in the discussion. The designers could annotate, move, duplicate, open or close the images displayed on the tabletop with their fingers. This demonstrated how the tabletop mediates cognition by facilitating visual access to other's drawings through sharing a common work space.

However, the designers were observed to use pen and paper most of the time when making changes or suggestions to the drawings rather than using the annotate feature in DiamondTouch. The designers had to press the annotate option every time they wanted to draw on the tabletop and the image produced from annotation would not reflect on the drawing permanently. Pressing the annotate option could had been cumbersome and the temporary image drawn could had led the designers to use the pen and paper for sketching. Researcher feels that the use of a stylus would be a preferred device for annotation in this task. As claimed by Wang and Blevis (2004), it is imperative that interaction technologies do not impede the dynamic nature of the interactions that involve sketching and annotation. The annotation feature in DiamondTouch could have been unsuitable or the designers would need more time to adapt to it. The time limit introduced could have also caused the designers sought to make their work as efficient as possible and taking very few risks while solving the problem. Nevertheless, this finding provides an awareness of the cognitive implications of changes to work processes and the potential weaknesses in the design of DiamondTouch or its prototype collaborative application.

\section{CONCLUSION}

The collaborative task in this study is the result of a social process to transform loosely defined design specifications towards a design solution. It involves the specification of work to shape the information processing activities that were applied in the problem resolution. The processes and the physical representation 
within the artifacts were critical to the problem solving. Researcher observed on the many ways a representation of the document was created, challenged, changed and harmonized as a final proposal was produced (Figure 7) by adapting the framework of distributed cognition. The shared context allows the creation, propagation and transformation of the design from one version to another. The framework also allowed researcher to examine the relationship between actors, artifacts, and the settings in which interactions occur. It allowed the researcher to identify characteristics which could enhance the usability of DiamondTouch, and also some potential weaknesses of the tabletop. The representations flowing through functional systems as objects of analysis could be identified and allowed researcher to reason about design artifacts and cognitive artifacts within a single conceptual framework.

There were interdependencies amongst the representational used which required all the design artifacts to be examined together as components of the process. The output of activity around the tabletop became the input of the activity at the personal computer and vice versa. As noted by Perry (1997), artifacts are not just partial representational steps towards a design solution, but are central to generating an understanding of the problem. In the observation and evaluation done, the used of DiamondTouch was seen able to facilitate distributed cognitive activities

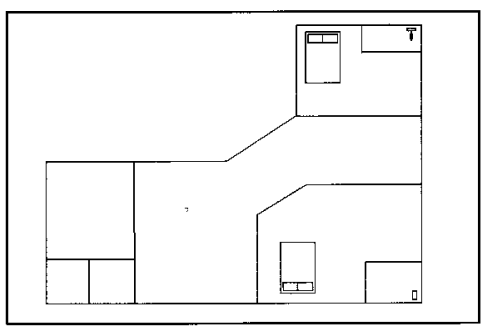

Version 1

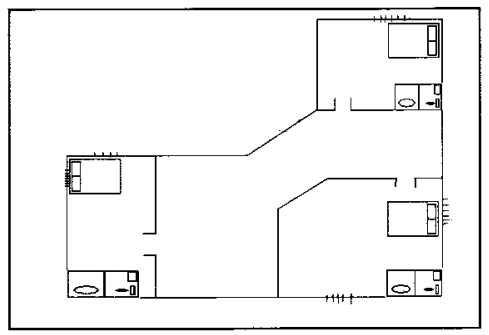

Version 2 among designers to generate design solution in this collaborative environment. The designers revealed various ways of recruiting the environment as their cognitive ally through the use of the tabletop and other artifacts. A detailed analysis on the collaborative activities around DiamondTouch is being conducted further to challenge or to support this established hypothesis.

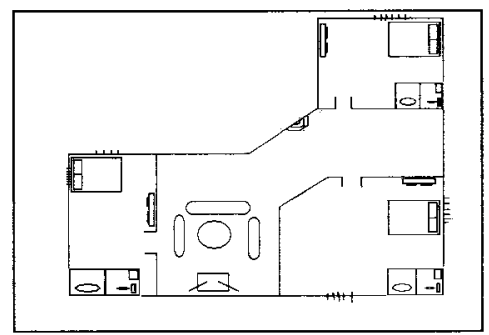

Version 3

Figure 7 : Three versions of drawing produced 


\section{REFERENCES}

Damianos, L., Hirschman, L., Kozierok, R., Kurtz, J., Greenberg, A., Walls, K., Laskowski, S., \& Scholtz, J. (1999). Evaluation for Collaborative Systems. ACM Computing Surveys (CSUR).

Dietz, P., \& Leigh, D. (2001). DiamondTouch: A Multi-User Touch Technology. UIST 2000.

Gutwin, C., and Greenberg, S. (1999) The effects of Workspace Awareness Support on the Usability of Real-Time Distributed Groupware. ACM Transactions on CHI, Vol 6(3).

Halverson, C. A. (1994). Distributed Cognition as a theoretical framework for HCI: don't throw the baby out with the bathwater - the importance of the cursor in air traffic control, COGSCI Tech Report 94-03. San Diego: Department of Cognitive Science, University of California, San Diego.

Hennessy S., \& Murphy, P. (1999). The Potential for Collaborative Problem Solving in Design and Technology. International Journal of Technology and Design Education., 9: 1-36.

Hussain, N., DeBruijn, O., Hassan, Z. (2005). Experimental Findings with Collaborative Interactions within a Co-Located Environment. ICITA2005, Sydney, Australia.

Hutchins, E. (1990). The Technology of Team Navigation. In J. R. Galegher, R. Kraut, \& L. Egido (Eds.), Intellectual Teamwork: Social and Technological Foundations of Cooperative Work: 191-220: Lawrence Erlbaum Associates.

Hutchins, E. (1995). Cognition in the Wild. Bradford: MIT Press.

Hollan, J., Hutchins, E., Kirsh, D. 2000. Distributed Cognition: Toward a New Foundation for Human-Computer Interaction Research. ACM Transactions on Computer-Human Interaction, vol. 7(2):174-196.

Nardi, B. A. (1995). Context and consciousness: Activity theory and Human-Computer Interaction. MTT Cambridge, MA, USA., pp 7-16.

Perry, M. J. (1997). Distributed Cognition and Computer Supported Collaborative Design: The organisation of work in Construction Engineering. Unpublished $\mathrm{PhD}$, Brunel, London.

Pinelle, D., Gutwin, C., Greenberg, S. (2004). Task Analysis for Groupware Usability Evaluation: Modeling Shared-Workspace Tasks with the Mechanics of Collaboration. ACM Transactions on Computer Human Interaction, vol. 10(4), 2004, 281-311.

Rogers, Y., Ellis, J., (1994). Distributed Cognition: an alternative framework for analysing and explaining collaborative working. Journal of Information Technology, vol 9(2): 119128.

Wang, H., \& Blevis, E. (2004). Concepts that Support Collocated Collaborative Work Inspired by the Specific Context of Industrial Designers. CSCW'04, Chicago, Illinois, USA. 